\title{
Frequência de comportamentos alimentares inadequados e sua relação com a insatisfação corporal em adolescentes
}

\author{
Frequency of abnormal eating behaviors and its \\ relationship with body dissatisfaction in adolescents
}

Tatiana Araújo Bertulino da Silva', Rosana Christine Cavalcanti Ximenes², Manuelle de Araújo Holanda3, Michel Gomes de Melo ${ }^{4}$, Everton Botelho Sougey ${ }^{5}$, Geraldo Bosco Lindoso Couto ${ }^{6}$

\section{RESUMO}

Objetivo: Descrever a relação entre a frequência de insatisfação com a imagem corporal e a presença de sintomas de transtornos alimentares em adolescentes. Métodos: $\bigcirc$ estudo foi realizado com uma amostra de 300 adolescentes, de ambos os sexos, na faixa etária de 10 a 17 anos, estudantes de uma escola pública estadual da cidade do Recife, Brasil. Foram utilizadas as versões brasileiras para adolescentes de três escalas autoaplicativas (EAT-26, BITE e $\mathrm{BSQ}$ ), além de um questionário contendo dados biodemográficos. Resultados: A frequência de sintomas de transtornos alimentares detectada pelo EAT-26 foi de 32,3\%; 2,3\% para comportamentos sugestivos de bulimia nervosa, por meio da escala BITE, tendo 36,67\% dos alunos apresentado padrão alimentar não usual; 5,6\% apresentaram insatisfação com a imagem corporal; 8,6\% demonstraram tendência à preocupação com a insatisfação com a imagem

\section{Palavras-chave}

Transtornos da

alimentação, insatisfação

corporal, adolescente.

\section{Keywords}

Eating disorders, body

dissatisfaction, adolescent. corporal e 17,6\% apresentaram leve insatisfação, além de 66,3\%, que mostraram normalidade em relação à sua forma corporal. Conclusão: Os adolescentes apresentaram níveis de alteração na autoimagem corporal, com alta frequência de insatisfação da imagem corporal, podendo apresentar associação com comportamentos alimentares inadequados.

\section{ABSTRACT}

Objective: To describe the relationship between frequency of body dissatisfaction and symptoms of eating disorders in adolescents. Methods: The study was conducted with a sample of 300 adolescents of both sexes, aged from 10 to 17 years-old, students from a state school in Recife, Brazil. Authors used the Brazilian version of three self-report questionnaires for adolescents (EAT-26, BITE and BSQ), and a questionnaire containing biodemographical data. Results: The frequency of symptoms of eating disorders detected by the EAT-26 was $32.3 \%$; $2.3 \%$ for suggestive behaviors of bulimia nervosa, through BITE, and $36.67 \%$ of the students presented an unusual eating pattern; $5.6 \%$ showed body dissatisfaction, and $66.3 \%$, showed normality in relation to its body form. Conclusion: These adolescents had higher levels of change in body self-image, with high frequency of body dissatisfaction, that suggest and association with inappropriate eating behaviors.

1 Universidade Federal de Pernambuco (UFPE), Programa de Pós-graduação em Neuropsiquiatria e Ciências do Comportamento. 2 UFPE, Núcleo de Enfermagem - Centro Acadêmico de Vitória (CAV), Programa de Pós-graduação em Neuropsiquiatria e Ciências do Comportamento.

3 UFPE, Fundação de Amparo à Ciência e Tecnologia do Estado de Pernambuco (Facepe).

Recebido em 27/1/2012 Aprovado em
$11 / 8 / 2012$

5 UFPE, Departamento de Neuropsiquiatria.

6 UFPE, Departamento de Clínica e Odontologia Preventiva. 


\section{INTRODUÇÃO}

A adolescência é uma fase em que ocorrem mudanças morfológicas, psicológicas e comportamentais, sendo a preocupação com o corpo muito evidente, a qual exerce forte influência sobre a saúde do indivíduo'. Um dos distúrbios comportamentais mais frequentes é o padrão alimentar inadequado, que tem implicações no crescimento e na saúde física e mental do jovem, além de não proporcionar subsídios para o total aproveitamento de sua potencialidade?2.

Os extremos de consumo alimentar podem caracterizar-se em desordens alimentares ou transtornos alimentares, apresentando-se a anorexia e a bulimia nervosa como principais disfunções da alimentação ${ }^{3}$. Na anorexia nervosa, ocorre uma busca incansável pela magreza, levando a pessoa a uma severa perda de peso. Para atingir tal finalidade, são usados os mais diferentes e bizarros recursos. Já o indivíduo bulímico faz ingestão compulsiva de grande quantidade de alimentos, seguida de vômito autoinduzido ou de abuso de laxantes e diuréticos ${ }^{4}$.

Os transtornos alimentares estão comumente associados à insatisfação com a imagem corporal que os adolescentes apresentam nessa etapa da vida. A imagem corporal é considerada como a forma que o corpo se apresenta para cada sujeito. Sua determinação é formada tanto em relação a si mesmo como em relação às outras pessoas 5 .

No decorrer de sua existência, o indivíduo cria diferentes imagens mentais sobre o seu corpo. Essas imagens são construídas e desconstruídas incessantemente ao longo de sua vida, a partir de suas observações objetivas e experiências emocionais, sociais e físicas acumuladas. Mesmo sendo importante a opinião alheia para a construção de uma imagem corporal, é a nossa concepção de nós mesmos que possui maior influência na criação da imagem mental que criamos de nosso corpo ${ }^{4,5}$.

A estima corporal e a insatisfação corporal são consideradas por alguns autores como os dois componentes constituintes da imagem corporal. A primeira inclui aspectos gerais da pessoa, como cabelo, rosto, pernas, peso e forma do corpo, ou seja, se refere ao aspecto global do corpo. Já a insatisfação corporal está diretamente ligada a preocupações com o peso, forma do corpo e gordura corporal. A insatisfação pode afetar aspectos da vida do indivíduo no que diz respeito ao seu comportamento alimentar, autoestima e desempenhos psicossocial, físico e cognitivo ${ }^{6}$.

A insatisfação com o corpo tem sido frequentemente ligada à discrepância entre a percepção e o desejo relativo a um tamanho e a uma forma corporal ${ }^{6}$. Em estudo prospectivo, foi demonstrado que a preocupação com o peso, a insatisfação corporal e a história de dieta no início da adolescência são fatores predisponentes para a presença de distúrbios alimentares nos anos subsequentes. Além disso, verificou-se que a insatisfação com o corpo está associada a baixa autoestima e limitações no desempenho psicossocial, associando-se também a quadros depressivos?

No entanto, existem poucos relatos de produções nacionais que investigaram essa associação, especialmente em população não clínica. Por essa razão, o objetivo deste estudo foi pesquisar a relação entre a insatisfação com a imagem corporal e a presença de sintomas de transtornos alimentares em adolescentes.

\section{MÉTODOS}

O estudo foi realizado com uma amostra de 300 adolescentes, de ambos os sexos, na faixa etária de 10 a 17 anos, estudantes de uma escola pública estadual da cidade do Recife, Brasil.

Essa amostra correspondeu ao total de $25 \%$ da amostra de um estudo maior, a dissertação intitulada "Prevalência de sintomas de transtornos alimentares e insatisfação corporal em jovens recifenses", que utilizou para a base de seu cálculo amostral a população de 85.701 estudantes regularmente matriculados no ensino público estadual, segundo dados da Secretaria de Educação do Estado de Pernambuco. Além disso, foram utilizados o erro de 3,0\%, a confiabilidade de $95,0 \%$ e a proporção esperada de $33,1 \%$, obtida no estudo de Ximenes et al.8. Para o cálculo, foi utilizado o programa Epi-Info, versão 6.04 para DOS. Por se tratar de uma amostra por conglomerados, o valor encontrado foi multiplicado por 1,2 , por conta do efeito cluster e aumentado em $20 \%$ para que perdas eventuais não comprometessem a representatividade da amostra.

Foram utilizadas as versões brasileiras para adolescentes de três escalas autoaplicativas. Para o rastreamento de sintomas de transtornos alimentares, foi selecionado o Teste de Atitudes Alimentares (EAT-26 - versão para adolescentes), cujas respostas fornecidas a cada questão pontuam entre 0 e 3, sendo a maior pontuação conferida à resposta extrema9,10. Os adolescentes que obtiveram o escore de 21 pontos ou mais no EAT-26 foram classificados como sintomáticos para transtornos alimentares, ou seja, com comportamento alimentar de risco para desenvolvimento do transtorno. Para rastreamento de sintomas bulímicos, foi utilizado o Teste de Avaliação Bulímica de Edimburgo (BITE - versão para adolescentes), o qual é composto por 33 questões de autopreenchimento, consistindo de duas subescalas de pontuação: a escala sintomática, para avaliar o grau de sintomas presentes, e a escala de gravidade, que fornece um índice da gravidade do comportamento compulsivo, definido pela frequência em que ocorrem. Um escore igual ou maior que 20 indica comportamento de compulsão alimentar com grande possibilidade de bulimia; quando ele se encontra entre 10 e 19, sugere-se um padrão alimentar não usual, necessitando avaliação por uma entrevista clínica ${ }^{11,12}$. 
A avaliação da imagem corporal foi realizada pelo Body Shape Questionnaire (BSQ), uma escala tipo Likert, em que as respostas fornecidas a cada questão pontuam entre 1 e 6 , sendo a maior pontuação conferida à resposta extrema na direção da insatisfação corporal ${ }^{13-15}$. O teste permite avaliar a preocupação com a insatisfação corporal, especialmente no sentido de sentir-se com excesso de peso. A classificação dos resultados foi realizada pelo total de escores obtidos e reflete os níveis de insatisfação corporal. Obtendo resultado menor ou igual a 80 pontos, é constatado um padrão de normalidade e tido como ausência de insatisfação corporal. Resultados entre 81 e 110 pontos são classificados como grau leve de insatisfação corporal. Já os resultados entre 111 e 140 são classificados como grau moderado de insatisfação corporal e, para resultados acima de 140 pontos, a classificação é de presença de grave grau de insatisfação corporal ${ }^{16}$.

Os questionários foram autoexplicativos, contendo questões de fácil interpretação, sendo as três escalas anexadas e entregues aos pesquisados, a fim de que pudessem responder de uma única vez a todos os questionários.

Todos os adolescentes responderam também a um questionário contendo dados biodemográficos.

Este projeto foi aprovado pelo comitê de ética em pesquisa com seres humanos da Universidade Federal de Pernambuco. Participaram do estudo todos os adolescentes cujos responsáveis assinaram ao termo de consentimento livre e esclarecido.

Na análise dos dados, foram utilizadas técnicas de estatística descritiva, mediante distribuições absolutas, percentuais e das medidas estatísticas: média, mediana e desvio-padrão. A margem de erro utilizada na decisão dos testes estatísticos foi de $5,0 \%$.

O programa utilizado para digitação e armazenamento dos dados foi o Excel 2010, e as análises foram realizadas no Bioestat 5.0.

\section{RESULTADOS}

As características observadas com frequência nos questionários foram: desejo de ser mais magro, intolerância ao excesso de peso e preocupação obsessiva com a comida.

A idade dos adolescentes pesquisados variou de $10 \mathrm{a}$ 17 anos, tendo média de 13,5 anos, mediana de 13,00 anos e desvio-padrão de 1,44 anos. A amostra populacional foi composta por $188(62,67 \%)$ adolescentes do sexo feminino e 112 (37,33\%) do sexo masculino. A frequência de sintomas de transtornos alimentares, detectada pelo EAT-26 no presente estudo, foi de 32,3\% (Gráfico 1).

A frequência de alunos com comportamentos sugestivos de bulimia nervosa por meio da escala BITE foi de 2,3\% (Tabela 1). Ainda em relação à mesma escala, no aspecto de sintomas, $36,67 \%$ dos alunos apresentaram escore entre 10-19, suges-

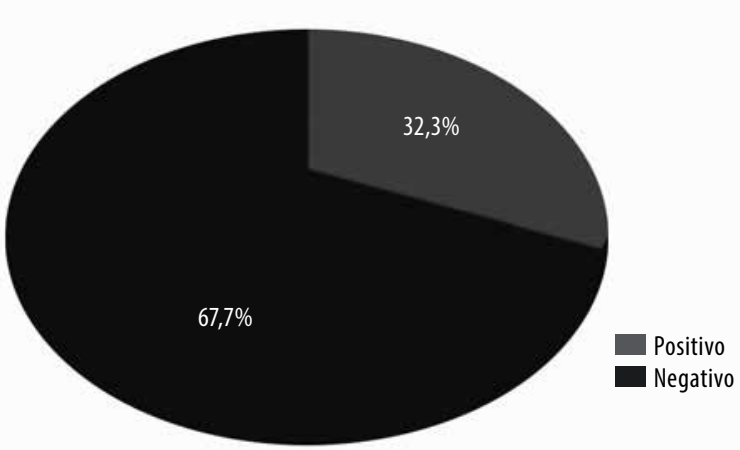

Gráfico 1. Distribuição do número de adolescentes pesquisados segundo os sintomas de transtornos alimentares (EAT-26).

tivo de padrão alimentar não usual. Dentre os pesquisados, $61,7 \%$ apresentaram um escore de até 5 pontos, estando eles dentro dos padrões de normalidade segundo a escala BITE.

Tabela 1. Avaliação da bulimia nervosa segundo o BITE

\begin{tabular}{lcc}
\hline BITE & F1 & $\%$ \\
\hline Médio & 110 & 36,67 \\
Grave & 7 & 2,33 \\
Ausente & 183 & 61,7 \\
Total & 300 & 100 \\
\hline
\end{tabular}

No grupo de adolescentes pesquisados foram encontrados estudantes que apresentavam algum grau de insatisfação com a imagem corporal, o qual foi evidenciado pelos seguintes fatores: descontentamento com a imagem corporal, preocupação com o peso e formas de perder peso nocivas à saúde.

No presente estudo, 5,6\% (17 casos) apresentaram insatisfação com a imagem corporal, 8,6\% (26 casos), tendência à preocupação com a insatisfação corporal e 17,6\% (53 casos), leve insatisfação, além de 66,3\% (199 casos), que mostraram normalidade em relação à sua forma corporal (Tabela 2).

Tabela 2. Avaliação da insatisfação da imagem corporal segundo o BSQ

\begin{tabular}{lcc}
\hline Insatisfaçäo & F1 & $\%$ \\
\hline Ausente & 204 & 68,0 \\
Leve & 53 & 17,6 \\
Média & 26 & 8,6 \\
Grave & 17 & 5,6 \\
Total & 300 & 100 \\
\hline
\end{tabular}

Percebe-se uma tendência à associação entre comportamento alimentar (EAT-26) e imagem corporal (BSQ). Foi verificado que, do total de avaliados, 32\% dos adolescentes possuem escores positivos para o EAT e alguma preocupação 
em relação à sua autoimagem corporal, nos níveis leve, moderado ou grave, o que sinaliza um índice expressivo de uma possível insatisfação com as imagens que têm de seus corpos.

\section{DISCUSSÃO}

Neste estudo, as adolescentes do sexo feminino mostraram que há uma tendência a apresentarem comportamento de maior risco para os transtornos alimentares, quando comparadas ao gênero masculino, independentemente do tamanho da amostra. Tais comportamentos são provenientes do medo mórbido de engordar, do constante desejo de perder peso e da insatisfação corporal ${ }^{17}$. Esse dado também foi encontrado no estudo de Gonçalves et al. ${ }^{18}$, quando avaliaram uma população de jovens que apresentavam riscos para o desenvolvimento de transtornos alimentares. Como a presença de anorexia na população geral é de 1\%, com 90\% dos casos em mulheres, e seu diagnóstico exige entrevista clínica, torna-se mais difícil avaliar grupos populacionais apenas por questionários autoexplicativos ${ }^{19}$.

A frequência de sintomas de transtornos alimentares no presente estudo foi de 32,3\%, semelhante ao resultado obtido por Pinto et al. ${ }^{20}$, que analisaram estudantes e observaram que cerca de $28 \%$ dos alunos possuíam risco elevado de apresentar transtornos alimentares, número tão expressivo quanto o encontrado pelo presente trabalho. Além do estudo realizado por Dunker et al. ${ }^{21}$, que também encontraram taxas significativas, cuja porcentagem de escore EAT-26 sugestivos de transtornos alimentares foi de 34,3\%. Quando foi referida a frequência de comportamento sugestivo de bulimia nervosa, foi observado um percentual de 2,3\% entre os adolescentes. Esse percentual encontrado é semelhante ao obtido por Souza et al.22, que encontraram, aproximadamente, 4\% de sintomas entre estudantes universitárias, as quais apresentaram padrão alimentar severamente perturbado e compulsão para comer, além de ser similar à faixa apontada pela literatura internacional de uma prevalência esperada entre $1 \%$ e $4 \%{ }^{23}$.

Em relação aos sintomas, 36,67\% dos adolescentes apresentaram características sugestivas de padrão alimentar não usual. No estudo de Cenci et al. ${ }^{24}$, o valor encontrado para a categoria comportamentos alimentares não usuais foi de 20,9\%. Assim como o presente estudo, ambos demonstraram que os valores sugestivos de padrão alimentar não usual tende a ser de 5 a 15 vezes maior que o padrão para valores sugestivos de bulimia nervosa.

Quando referido aos padrões de normalidade, que é um escore de até 5 pontos, obteve-se uma frequência de 61,7\%. Em um estudo publicado por Mehler ${ }^{25}$, foi demonstrada uma distribuição de ocorrências entre mulheres e homens de 10:1 a 20:1, e o índice encontrado no presente estudo foi de 1:1, demonstrando que a frequência de bulimia nervosa entre mulheres e homens pode estar aumentando no decorrer das décadas. No entanto, seria necessário um estudo populacional com maior representatividade para que esses dados sejam afirmados com relação à população da cidade do Recife.

A insatisfação com a imagem corporal entre os adolescentes que participaram da pesquisa foi de 5,6\% (17 casos). Atualmente, existe pouca dúvida de que o mais prevalente aspecto da insatisfação dos adolescentes em relação a seu peso e aparência é a crença de que eles estão acima do peso, mesmo quando têm seu peso eutrófico ou abaixo do recomendado. Sugere-se que a pressão social seja responsável pelas preocupações acerca da imagem corporal nesses adolescentes $^{26}$. Há evidências de que os meios de comunicação podem contribuir para uma maior insatisfação com a imagem corporal, exacerbando dificuldades naturais de aceitação do próprio corpo, na medida em que transmitem imagens e padrões estéticos centrados na magreza como ideal de beleza ${ }^{27}$.

Do total de avaliados, 32\% dos adolescentes possuíam escores positivos para o EAT, corroborando com o estudo de Bosi et al. ${ }^{28}$, em que a população estudada apresentou escore do BSQ de 33,6\%, demonstrando a insatisfação corporal. Ao cruzar os resultados de ambos os instrumentos, verificou-se que $11 \%$ dos pesquisados apresentaram sintomas de transtornos alimentares ligados à insatisfação da imagem corporal.

Embora haja presença de insatisfação com a imagem corporal na população estudada, é necessário destacar que entre os escolares do gênero feminino há maior prevalência. Além disso, quando feita a análise estratificada, ou seja, nos diversos graus de preocupação, evidencia-se que os escolares do sexo feminino igualmente responderam aos graus (leve, moderado e grave) com valores de 60\%, enquanto o masculino apresentou um valor de 40\%.

\section{CONCLUSÃO}

Os dados obtidos na presente pesquisa, juntamente com os da literatura, permitem concluir que os adolescentes avaliados apresentaram níveis de alteração na autoimagem corporal, com alta frequência de insatisfação da imagem corporal. Este trabalho pode indicar uma possível associação entre insatisfação da imagem corporal e comportamentos alimentares inadequados, visto a forte influência que os conceitos sobre imagem corporal desempenham no desenvolvimento de comportamentos sugestivos de transtornos alimentares.

As alterações na percepção corporal geram uma insatisfação que pode levar a comportamentos alimentares inadequados, com prejuízos no desenvolvimento à saúde, além de risco de desenvolvimento de transtornos alimentares. Tendo conhecimento sobre isso, faz-se necessário o desenvolvimento de campanhas e programas que estimulem a 
aceitação corporal e a adoção de hábitos alimentares e de vida saudáveis. É interessante também reduzir a influência da mídia, de modo a desvincular a imagem do corpo magro à atratividade e ao padrão de beleza ideal.

\section{AGRADECIMENTOS}

Agradecimentos à Coordenação de Aperfeiçoamento de Pessoal de Nível Superior (Capes) e à Fundação de Amparo à Ciência e Tecnologia do Estado de Pernambuco (Facepe).

Não houve conflitos de interesses.

Esta pesquisa trata-se do estudo piloto da dissertação de mestrado: "Prevalência de transtornos alimentares e insatisfação corporal em recifenses".

\section{REFERÊNCIAS}

1. Chemin C, Milito F. Transtornos alimentares em adolescentes. Rev Bras Obes Nutr Emagrecimento. 2007;1(2):84-8.

2. Fleitlich BW, Larino MA, Cobelo A, Cordás TA. Anorexia nervosa na adolescência. J Pediatr (Rio J). 2006;76(3):323-9.

3. Traebert J, Moreira EAM. Transtornos alimentares de ordem comportamental e seus efeitos sobre a saúde bucal na adolescência. Pesqui Odontol Bras. 2001;15(4):359-63.

4. Grando LH. Representações sociais e transtornos alimentares: as faces do cuidar em enfermagem [dissertação]. São Paulo: Escola de Enfermagem da Universidade de São Paulo; 2000.

5. Russo R. Imagem corporal: construção através da cultura do belo. Mov Percepção. 2005;5(6):80-90.

6. Almeida G, Santos JE, Passian SR, Loureiro SR. Percepção de tamanho e forma corporal de mulheres: estudo exploratório. Psicol Estud. 2005;10(1):27-35.

7. Triches RM, Giugliani ERJ. Insatisfação corporal em escolares de dois municípios da região Sul do Brasil. Rev Nutr. 2007;20(2):119-28.

8. Ximenes RCC, Couto G, Sougey E. Eating disorders in adolescents and their repercussions in oral health. Int J Eat Disord. 2010:43(1):59-64

9. Nunes MA, Bagatini L, Abuchaim AL. Considerações sobre o Teste de Atitudes Alimentares (EAT-26). Rev ABP-APAL. 1994;16:7-10.

10. Bighetti F, Santos CB, Santos JE, Ribeiro RPP. Tradução e validação do Eating Attitudes Test em adolescentes do sexo feminino de Ribeirão Preto-SP. J Bras Psiquiatr. 2004;53:339-46.
11. Cordás TA, Hochgraf PB. 0 BITE. Instrumento para avaliação da bulimia nervosa: Versão para o português. J Bras Psiquiatr. 1993;42:141-4.

12. Ximenes RCC, Colares V, Bertulino T, Couto GBL, Sougey EB. Versão brasileira do "BITE" para uso em adolescentes. Arq Bras Psicol. 2011;63(1):52-63.

13. Cooper PJ, Taylor MJ, Cooper M, Fairburn CG. The development and validation of the Body Shape Questionnaire. Int J Eat Disord. 1987;6:485-94.

14. Conti MA, Cordás TA, Latorre MRDO. A study of the validity and reliability of the Brazilian version of the Body Shape Questionnaire (BSQ) among adolescents. Rev Bras Saude Mater Infant. 2009:9(3):437-44.

15. Alves E, Vasconcelos FAG, Calvo MCM, Neves J. Prevalência de sintomas de anorexia nervosa e insatisfação com a imagem corporal em adolescentes do sexo feminino do Município de Florianópolis, Santa Catarina, Brasil. Cad Saude Publica. 2008;24(3):503-12.

16. Freitas S. Transtornos alimentares: introdução, diagnóstico, epidemiologia e etiologia. In: Brasil MAA, Botega NJ. PEC- Programa de Educação Continuada: Título de especialização em Psiquiatria 2000-2003. Rio de Janeiro: Guanabara Koogan; 2004. p. 196-204.

17. Fiates GMR, Salles RK. Fatores de risco para o desenvolvimento de distúrbios alimentares: um estudo em universitárias. Rev Nutr. 2001;14:3-6.

18. Gonçalves TD, Barbosa MP, Rosa LCL, Rodrigues AM. Comportamento anoréxico e percepção corporal em universitários. J Bras Psiquiat. 2008;57(3):166-70.

19. Assunção SSM, Cordás TA, Araújo LASB. Atividade física e transtornos alimentares. Rev Psiq Clin. 2002;19(1):4-13.

20. Pinto ACM, Camargo MR, Novo NF, Hübner CK. Transtornos alimentares em alunas da Faculdade de Medicina do Centro de Ciências Médicas e Biológicas da PUC-SP. Rev Fac Cienc Med Sorocaba. 2009;1:16-20.

21. Dunker KLL, Fernandes CPB, Carreira Filho D. Influência do nível socioeconômico sobre comportamentos de risco para transtornos alimentares em adolescentes. J Bras Psiquiat. 2009;58(3):156-61.

22. Souza FGM, Martins MCR, Monteiro FCC, Menezes Neto GC, Ribeiro IB. Anorexia e bulimia nervosa em alunas da Faculdade de Medicina da Universidade Federal do Ceará - UFC. Rev Psiq Clin. 2002;29(4):172-80.

23. Vale AMO, Kerr LRS, Bosi MLM. Comportamentos de risco para transtornos do comportamento alimentar entre adolescentes do sexo feminino de diferentes estratos sociais do Nordeste do Brasil. Ciênc Saúde Coletiva. 2011;16(1):121-32.

24. Cenci M, Peres KG, Vasconcelos FAG. Prevalência de comportamento bulímico e fatores associados em universitárias. Rev Psiq Clin. 2009;36(3):83-8.

25. Mehler PS. Bulimia nervosa. N Engl J Med. 2003;349:9.

26. Pesa JA, Syre TR, Jones E. Psychosocial differences associated with body weight among female adolescents: the importance of body image. J Adolesc Health. 2000;26:330-7.

27. Reato LFN. Mídia x Adolescência. Pediatr Mod. 2001;37:37-40.

28. Bosi MLM, Raggio LR, Morgado CMC, Costa MLSS, Carvalho RJ. Autopercepção da imagem corporal entre estudantes de nutrição: um estudo no município do rio de janeiro. J Bras Psiquiatr. 2006;55(2):108-13. 